\title{
Depredación pre-dispersiva de semillas en tres poblaciones del árbol Enterolobium contortisiliquum (Fabaceae)
}

\author{
Marcelo Nahuel Morandini \& Marta Leonor de Viana ${ }^{1}$ \\ 1. INEAH (Instituto de ecología y ambiente humano), Universidad Nacional de Salta, Avenida Bolivia 5150, Salta CP \\ 4400, Argentina; nahuelmorandini@hotmail.com; mldeviana@yahoo.com.ar
}

Recibido 21-VIII-2008. C Corregido 22-II-2009. Aceptado 25-III-2009.

\begin{abstract}
Pre-dispersal seed predation in three populations of the tree Enterolobium contortisiliquum (Fabaceae). Predation is an important selective force that has shaped different strategies related to size, quantity and production time of fruits, seeds and seed reserves. The seeds of E. contortisiliqum are predated by the bruchid Merobruchus bicoloripes. We collected 1300 fruits from 26 trees in San Salvador de Jujuy (Jujuy province), Castañares, and San Lorenzo (Salta province) Argentina. San Lorenzo is the wettest and highest site, while Castañares is the driest and lowest site. Jujuy is in the middle. In the laboratory, the fruits from each site were weighted and divided in three groups (small, medium and big). We counted the mature and predated seeds per fruit. In San Lorenzo, seed predation was higher in medium sized fruits while in Castañares and Jujuy seed predation was higher in both medium and bigger fruits. However, only in San Lorenzo we found a significant difference in preference for medium size fruits. Rev. Biol. Trop. 57 (3): 781-788. Epub 2009 September 30.
\end{abstract}

Key words: Pre-dispersal seed predation, bruchids, Enterolobium contortisiliquum, Merobruchus bicoloripes, San Salvador de Jujuy, Castañares, San Lorenzo, Argentina.

La depredación de semillas es considerada una fuerza selectiva importante ya que puede disminuir el éxito reproductivo de las plantas y afectar su reclutamiento y demografía, influyendo directa e indirectamente en el tamaño, la cantidad y el tiempo de producción de frutos y semillas (Janzen 1969, Mitchell 1977, Silvertown \& Lovett Doust 1993, de Viana 1999).

Chambers \& MacMahon (1994) clasificaron la depredación de frutos y semillas como pre y postdispersiva. La primera ocurre cuando las semillas aún se encuentran en la planta madre, por lo tanto, la muerte de la semilla se produce antes de su dispersión por agentes activos, afectando en forma directa el número de semillas que serán dispersadas. La postdispersiva afecta el establecimiento de las plántulas, debido a que las semillas ya dispersadas mueren antes de germinar. La depredación predispersiva es realizada principalmente por coleópteros, dípteros, lepidópteros e himenópteros, mientras que en la postdispersiva intervienen organismos más grandes como los pequeños mamíferos aunque las hormigas son también depredadores postdispersivos importantes (Janzen 1971, 1980, Crawley 1992, Moles et al. 2003).

Los coleópteros de la familia Bruchidae se han especializado en la depredación de semillas de Fabaceae. Las hembras de vida libre oviponen sobre o cerca de los frutos. Cuando los huevos eclosionan, las larvas ingresan a través de la pared del pericarpo, se introducen en una semilla, donde pasan por los diferentes estadios larvales. Completan su ciclo vital consumiendo una o varias semillas y emergen del fruto como adultos (Janzen 1969, Mitchell 1977, Terán \& Muruaga 1981). Los frutos pueden ser infectados cuando se encuentran en la planta madre aunque en algunos casos puede 
ocurrir re-infección con posterioridad a la etapa de dispersión. La depredación de semillas por bruquidos puede ocurrir en la fase predispersiva, por ejemplo en Cercidium floridum y varias especies del género Acacia (Mitchell 1977), o en ambas (pre y postdispersiva), como ocurre en Prosopis ferox (Ortega Baes et al. 2001).

Entre los mecanismos que pueden disminuir la depredación por brúquidos, se encuentran la presencia de testas seminales duras, la variabilidad en el tamaño de los frutos y el tamaño y cantidad de semillas por fruto (Credland 1990). El tamaño de los frutos puede constituir una fuente de atracción para las hembras ya que un tamaño mayor de fruto, ofrecería un paquete con mayor cantidad potencial de recursos, lo que garantizaría el desarrollo de las larvas. Por ejemplo, en $P$. ferox se encontró que los frutos de mayor tamaño producen semillas de mayor peso. Sin embargo, si la preferencia de los brúquidos es por los frutos de mayor tamaño, puede ocurrir que la competencia entre las larvas aumente por una mayor tasa de ataque en estos frutos. Estos y otros factores relacionados con la adecuación (fitness) biológica, tanto de las plantas como de los brúquidos, tendrán un efecto en la variabilidad de frutos y semillas.

El propósito de este trabajo fue estudiar la variación dentro y entre poblaciones de Enterolobium contortisiliqum (Vell) Morong (Fabaceae: Mimosoideae) en el peso de los frutos, el número de semillas por fruto (viables, depredadas y total) y en la preferencia por parte de los bruquidos, por algún tamaño de fruto en la etapa predispersiva. Para esto se consideraron tres poblaciones de E. contirtisiliquum, distribuidas en las provincias de Salta (Castañares \& San Lorenzo) y en la de Jujuy (San Salvador de Jujuy) en el Noroeste de Argentina.

\section{MATERIALES Y MÉTODOS}

E. contortisiliquum, es una especie arbórea nativa de amplia distribución en el norte argentino, de gran porte e inerme. Cuando crece en ambientes húmedos puede superar los $20 \mathrm{~m}$ de altura y el metro de diámetro. Posee hojas alternas, caducas, compuestas bipinnadas. Las inflorescencias son globosas, con flores perfectas de coloración blanca verdosa. La floración ocurre desde octubre a diciembre, los frutos son vainas achatadas indehiscentes, de forma arriñonada, de color negro a la madurez. La fructificación es de enero a abril. La fase dispersiva comienza en julio, aunque los frutos pueden permanecer hasta un año en la planta (Digilio \& Legname 1982, Dimitri et al. 1997).

Los frutos contienen numerosas semillas ovaladas de alrededor de $1 \mathrm{~cm}$ de largo, de tegumento liso muy duro y son de color castaño (Dimitri et al. 1997). La corteza y los frutos contienen taninos y saponinas. La madera es blanca liviana y flexible y se utiliza en la fabricación de cajones de frutas, colmenas, ventanas, puertas y marcos (Burkart 1943, Leonardis 1977).

Las semillas son depredadas por el brúquido M. bicoloripes pic (Muruaga com. person.). Las hembras oviponen principalmente en los folíolos, aunque también son sitios de oviposición los pecíolos, los raquis y la base de los frutos. Los huevos son colocados en grupos de 2 a11. Una vez ubicada la larva en el interior de la semilla, se alimenta de los cotiledones, dejando únicamente el tegumento. Esta operación puede repetirse con tres semillas más hasta llegar al estado de pupa, lo que depende del tamaño y la madurez de las semillas. Si la postura se efectúa sobre los frutos maduros, el brúquido consume sólo dos semillas hasta completar el estadio larval. Para completar el ciclo de larva a adulto son necesarios 90 días (Terán \& Muruaga 1981).

Entre abril y mayo de 2003, recolectamos 1300 frutos de 26 árboles en tres zonas: Castañares y San Lorenzo en la provincia de Salta (6 árboles, 50 frutos por árbol; 10 árboles, 50 frutos por árbol respectivamente) y en San Salvador de Jujuy (10 árboles, 50 frutos por árbol). Para cada zona determinamos la clasificación climática de acuerdo al balance hídrico según Thornthwaite (Strahler 1986). En el laboratorio, los frutos se pesaron con balanza analítica (0.001 g de precisión) y se extrajeron las semillas en forma manual, clasificándolas 
en viables y depredadas. Para estudiar si existe alguna relación entre el tamaño de los frutos y la depredación, los frutos de cada sitio se dividieron en tres clases de tamaño: chicos, medianos y grandes en base al peso. Los medianos fueron aquellos que se agruparon alrededor de la media \pm 1.5 error estándar. Mientras, que los chicos y grandes se concentraron en los valores extremos, de manera de poder contrastar las tres clases de tamaño, evitando continuidad en los pesos de una clase a otra. Para cada clase de tamaño, se seleccionaron 30 frutos de la población de San Lorenzo y 50 frutos de las poblaciones de Castañares y Jujuy. La preferencia se estimó como semillas depredadas / disponibilidad de semillas, para cada fruto y clase de tamaño. La preferencia por los tamaños de fruto se analizó dentro y entre sitios con Kruskal Wallis (KW). Todas las pruebas estadísticas se realizaron con Systat (1992) para Windows.

\section{RESULTADOS}

Clasificación climática. Los sitios de estudio difieren en altitud y parámetros climáticos, siendo San Lorenzo el más húmedo, de mayor altitud, con suelos desarrollados, pendientes pronunciadas (superiores al 5\%) y vegetación correspondiente a bosque montano con elementos de Yungas y Chaco. Castañares es el sitio de menor altitud, más seco, con suelos de incipiente desarrollo (apoyados sobre depósitos de río) y con vegetación de la Provincia Fitogeográfica Chaqueña. San Salvador de Jujuy presenta características intermedias (Cuadro 1).

Variabilidad y depredación intra-poblacional. En San Lorenzo, el peso promedio de los frutos fue de $16 \mathrm{~g}$ (mínimo $1.2 \mathrm{~g}$ y máximo $56 \mathrm{~g})$. El número promedio de semillas $( \pm$ S.E) por fruto fue de $13.0 \pm 0.6 \mathrm{y}$ el número de semillas depredadas por fruto de $2.0 \pm 0.3$. La depredación fue significativamente mayor en los frutos medianos (KW $=25 ; \mathrm{p}<0.0005)$.

En Castañares, el peso promedio de los frutos fue de $10 \mathrm{~g}$ (mínimo $1.2 \mathrm{~g}$ y máximo $30 \mathrm{~g}$ ). El número promedio de semillas por fruto fue de $12.0 \pm 0.3$ y el número de semillas depredadas por fruto de $4.0 \pm 0.2$. La depredación fue mayor en frutos medianos y grandes.

En San Salvador de Jujuy, el peso promedio de los frutos fue de $17 \mathrm{~g}$, también con gran variabilidad (mínimo $4 \mathrm{~g}$ y máximo $67 \mathrm{~g}$ ). El número promedio de semillas por fruto fue de $13.0 \pm 0.2$ y el número de semillas depredadas por fruto de $5.0 \pm 0.2$. Al igual que en castañares, la depredación fue mayor en frutos medianos y grandes (Cuadro 2).

$\mathrm{Si}$ bien la cantidad promedio de semillas por fruto fue similar en los tres sitios, encontramos diferencias significativas en todas las variables estudiadas: número de semillas viables, depredadas, número de semillas por fruto

CUADRO 1

Datos geoclimáticos de los sitios de estudio (altitud, coordenadas, precipitación y temperatura)

TABLE 1

Geoclimatic data (altitude, coordinates, precipitation and temperature) per study site

$\begin{array}{lccccc}\text { Localidad } & \begin{array}{c}\text { Altitud } \\ \text { m.s.n.m }\end{array} & \text { Ubicación } & \begin{array}{c}\text { Clasificación } \\ \text { climática }\end{array} & \begin{array}{c}\text { Precipitación media } \\ \text { anual }(\mathrm{mm})\end{array} & \begin{array}{r}\text { Temperatura } \\ \text { Media anual }\end{array} \\ \text { San Lorenzo } & 1487 & 65^{\circ} 31^{\prime} \mathrm{W}-24^{\circ} 11^{\prime} \mathrm{S} & \text { Húmedo } & 1395 \pm 237 & 16,73 \pm 4,19 \\ \text { Castañares } & 1173 & 65^{\circ} 25^{\prime} \mathrm{W}-24^{\circ} 47^{\prime} \mathrm{S} & \text { Sub-húmedo seco } & 678 \pm 131 & 16,72 \pm 4,19 \\ \text { S. S. de Jujuy } & 1259 & 65^{\circ} 18^{\prime} \mathrm{W}-24^{\circ} 11^{\prime} \mathrm{S} & \text { Sub-húmedo húmedo } & 826 \pm 230 & 16.46 \pm 3.9\end{array}$

La clasificación climática es según Thornthwaite.

Climatic classification by Thornthwaite. 
CUADRO 2

Número de semillas viables y depredadas (promedio \pm error estándar) por clase de tamaño de los frutos y peso promedio de los frutos de los sitios de estudio

TABLE 2

Number of viable and predated seeds (average \pm standard error) by fruit size and average weight of the fruits of the study sites

$\begin{array}{lccccc}\text { Sitio } & \begin{array}{c}\text { Tamaño de } \\ \text { los frutos }\end{array} & \begin{array}{c}\text { Peso }(\mathrm{g}) \\ \text { de los frutos }\end{array} & \text { Preferencia } & \begin{array}{c}\text { Número de } \\ \text { semillas viables }\end{array} & \begin{array}{c}\text { Número de semillas } \\ \text { depredadas }\end{array} \\ \text { Sequeños } & 3.6 \pm 0.2 & 0.06 \pm 0.02 & 6.7 \pm 0.7 & 0.4 \pm 0.1 \\ & \text { Medianos } & 12.1 \pm 0.04 & 0.26 \pm 0.04 & 10.2 \pm 0.6 & 3.8 \pm 0.5 \\ \text { Gastañares } & \text { Grandes } & 32.8 \pm 1.3 & 0.09 \pm 0.03 & 16 \pm 0.7 & 1.37 \pm 0.4 \\ & \text { Pequeños } & 4.5 \pm 0.1 & 0.3 \pm 0.04 & 5.8 \pm 0.6 & 2.1 \pm 0.2 \\ \text { Medianos } & 10.5 \pm 0.1 & 0.37 \pm 0.03 & 8.8 \pm 0.6 & 4.9 \pm 0.4 \\ \text { S.S. de Jujuy } & \text { Grandes } & 18.6 \pm 05 & 0.37 \pm 0.03 & 10 \pm 0.6 & 5.5 \pm 0.4 \\ & \text { Pequeños } & 6.9 \pm 0.1 & 0.33 \pm 0.04 & 6.9 \pm 0.6 & 3.2 \pm 0.4 \\ & \text { Medianos } & 16.5 \pm 0.1 & 0.36 \pm 0.04 & 8.4 \pm 0.7 & 4.7 \pm 0.5\end{array}$

$\mathrm{y}$ peso de los frutos (KW; $\mathrm{p} \leq 0.05$ en todos los casos) (Fig. 1).

Preferencia: En San Lorenzo, la preferencia fue significativamente mayor en los frutos de tamaño medio ( $\mathrm{KW}=25 ; \mathrm{p}<0.0005)$, mientras que en Castañares y en San Salvador de Jujuy, no se encontraron diferencias significativas en la preferencia de los brúquidos por alguna clase de tamaño (Cuadro 2, Fig. 2).

\section{DISCUSIÓN}

Las producción de semillas puede variar espacial y temporalmente, pudiendo estar limitada por la cantidad de óvulos producidos, la cantidad y calidad del polen transferido, la cantidad de nutrientes y fotosintatos disponibles para los frutos y semillas, enfermedades y agentes del ambiente físico (Silvertown \& Lovett Doust 1993, de Viana 1995).

A pesar de estar relacionados directamente con la adecuación biológica, la producción de frutos y semillas muestran amplias variaciones tanto entre poblaciones de la misma especie como entre individuos de la misma población y aún en un mismo individuo (Wulff 1986).
Suele ser común que las plantas pierdan por depredación entre el 90 y $100 \%$ de una producción dada (Crawley 1992). Moles et al. (2003) reportaron una supervivencia predispersiva del $55 \%$ y postdispersiva del $50 \%$ en una variedad de especies. En E. contortisiliquum, la supervivencia predispersiva fue diferente en los sitios estudiados, alcanzando un máximo de $86 \%$ en la población de San Lorenzo y mínimos similares en la de Castañares (65\%) y San Salvador de Jujuy (66\%).

La proporción de semillas depredadas por fruto en Castañares y en Jujuy son similares a las reportadas por Terán y Muruaga (1981) para la misma especie. La menor proporción de semillas depredadas en San Lorenzo podría deberse a una menor abundancia del brúquido $M$. bicoloripes en este sitio que es mucho más húmedo $\mathrm{y}$ de mayor altitud. Sin embargo esto debería ser corroborado en futuros estudios.

En San Lorenzo la depredación predispersiva es mayor en los frutos medianos. En la medida en que aumenta el nivel de depredación, la preferencia por alguna clase de tamaño de frutos disminuiría como consecuencia de la competencia entre brúquidos por los frutos del tamaño preferido. Esto se refleja en Castañares 

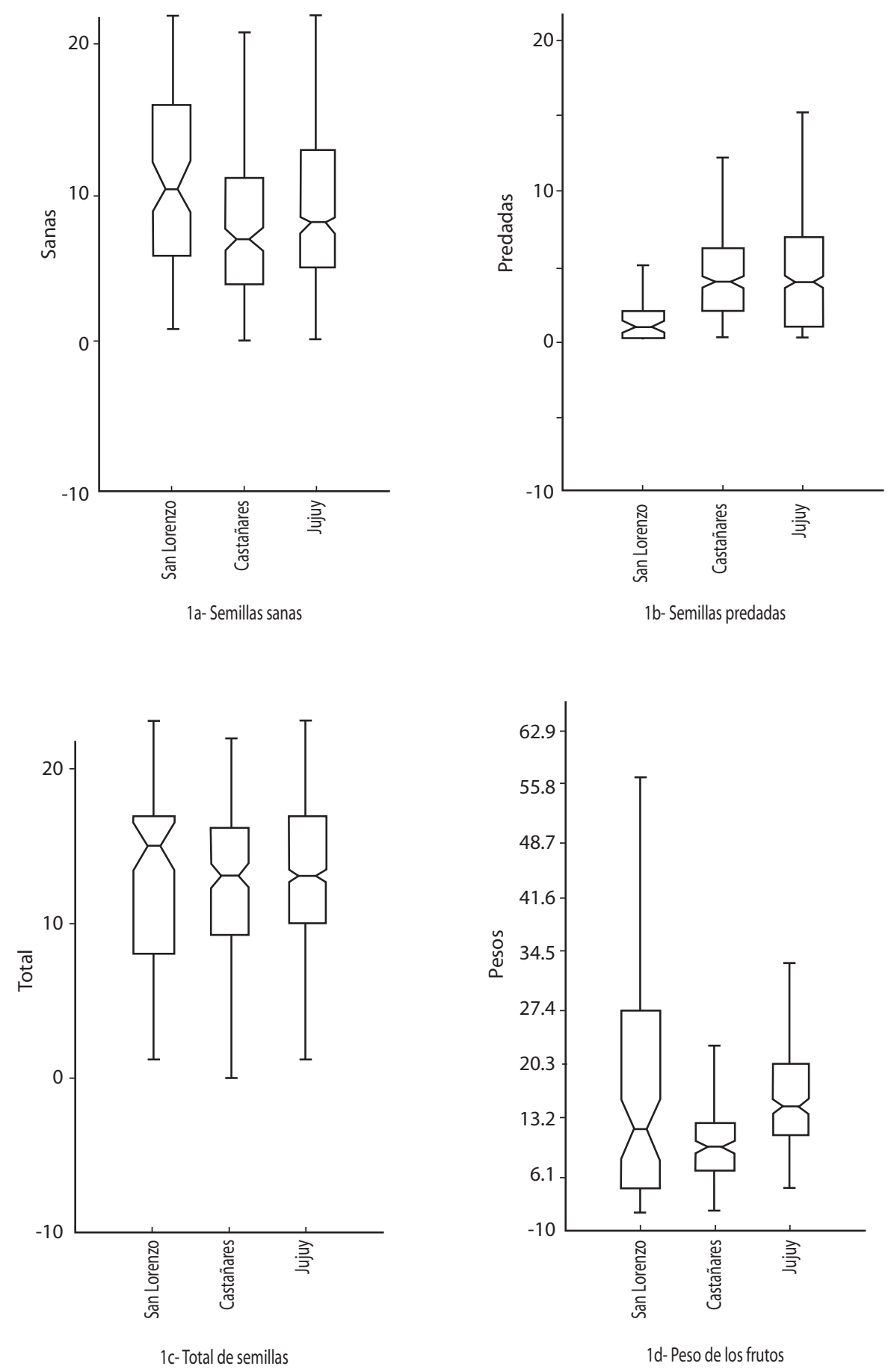

Fig. 1. Número de semillas viables, depredadas y total de semillas por fruto y peso de los frutos ( $g$ ) en los tres sitios de estudio. En todos los casos las diferencias entre sitios son significativas ( $\mathrm{KW} ; \mathrm{p} \leq 0.05)$.

Fig. 1. Number of total, viable and predated seeds per fruit and fruit weight (g) in the three study sites. In all cases, the differences among sites are significant $(\mathrm{KW} ; \mathrm{p} \leq 0.05)$. 

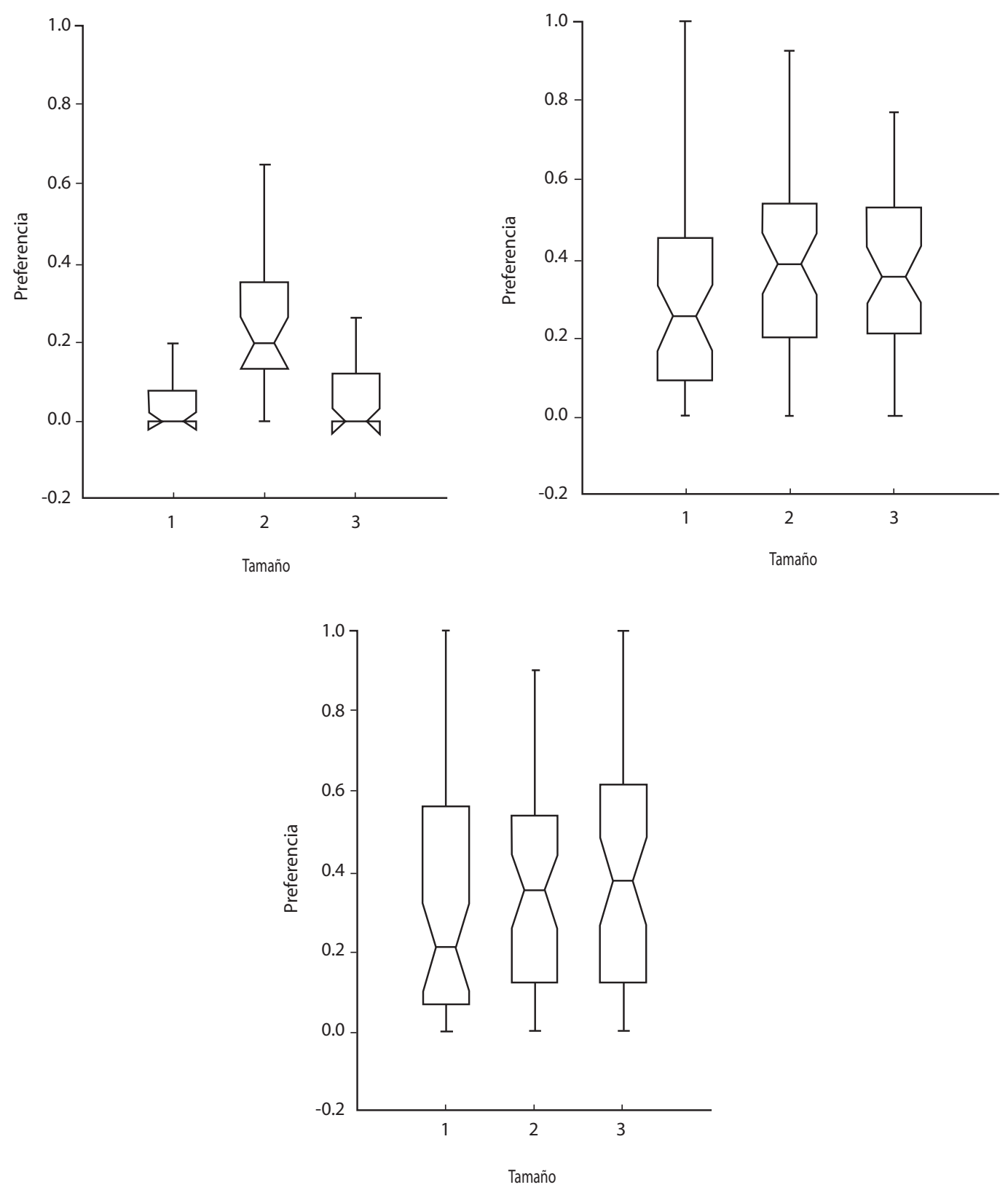

Fig. 2. Preferencia por distintas clases de tamaño de los frutos de E. contortisiliquum en San Lorenzo (a), Castañares (b), y Jujuy (c). Tamaño del fruto 1: pequeño; 2: mediano; 3: grande.

Fig. 2. Preference for different size class of E. contortisiliquum fruits in San Lorenzo (a), Castañares (b), and Jujuy (c). Fruit size 1: small; 2: medium; 3: large. 
y Jujuy (con mayores niveles de depredación) donde no existen diferencias significativas en preferencia entre las clases de tamaño consideradas. Sin embargo aquí habría que suponer que el tamaño de las semillas es similar en las distintas clases de tamaño de los frutos y esta es una característica que no se abordó en este trabajo, aunque otros autores no encontraron relaciones definidas entre el peso de las semillas y la supervivencia pre y post-dispersiva (Moles et al. 2003).

\section{AGRADECIMIENTOS}

Agradecemos a María José Trivisono, Federico Coronado, Diego Galagovsky y Marín Ibarra que trabajaron en la recolección y procesado de los frutos. A Susana Muruaga De L' Argentier por su aporte en la determinación del brúquido y de material bibliográfico, nuestro profundo agradecimiento.

\section{RESUMEN}

La depredación se ha postulado como una importante fuerza selectiva que ha moldeado distintas estrategias relacionadas con el tamaño, la cantidad y el tiempo de producción de semillas, frutos y el contenido de reservas. Las semillas de Enterolobium contortisiliqum son depredadas por el brúquido Merobruchus bicoloripes. Se estudiaron tres poblaciones de E. contortisiliquum en relación al tamaño de los frutos, a la producción de semillas por fruto, y a la depredación de semillas, comparando la variabilidad dentro y entre poblaciones. Se recolectaron 1300 frutos de 26 árboles en San Salvador de Jujuy (Jujuy), Castañares y San Lorenzo (Salta). Los frutos se pesaron y se contaron las semillas viables y depredadas. San Lorenzo es el sitio más húmedo y de mayor altitud, y Castañares el de menor altitud y más seco. En San Lorenzo encontramos una mayor preferencia de depredación en frutos medianos. En Castañares y San Salvador de Jujuy, a pesar de que los frutos medianos y grandes presentaron mayores niveles de depredación, no encontramos diferencias en la preferencia por alguna clase de tamaño. Los mayores niveles de depredación se encontraron en Castañares y S. S. de Jujuy.

Palabras clave: depredación pre-dispersiva, brúquidos, Enterolobium contortisiliquum, Merobruchus bicoloripes, San Salvador de Jujuy, Castañares, San Lorenzo, Argentina.

\section{REFERENCIAS}

Bawa, K.S. \& C.J. Webb. 1984. Flower, fruit and seed abortion in tropical for the evolution of paternal and maternal reproductive patterns. Am. J. Bot. 71: 736-751.

Bianchi, A.R. \& C.E. Yañez. 1992. Las precipitaciones en el Noroeste Argentino. Segunda Edición, INTA. Salta. Argentina.

Chambers J.C. \& J.A. Mac Mahon 1994. A day in the life of a seed: Movements and fates of seeds and their implications for natural and managed systems. Annu. Rev. Ecol. Syst. 25: 263-292.

Cavers, P. B. \& M. G. Steel 1984. Patterns of change in seed weight over time on individual plants. Am. Nat. 124: 324-335.

Crawley, M.J. 1992. Seed predators and plant population dynamics. In: Fenner, M. (Ed). Seeds: the ecology of regeneration in plant communities. $\mathrm{CAB}$ International, Wallingfor, Oxfordshire, Inglaterra.

Creadland, P.F. 1990. Biotype variation and host change in bruchids: causes and effects in the evolution of bruchid pests. En: Fujii, K., Gatehouse, A.M., Johnson, C.D., Mitchell, R \& Yosida, T. (eds). Bruchids and legumes: economics, ecology and coevolution. Kluwer, Dordetch. Holanda.

de Viana, M.L. 1995. Distribución del cardón (Trichocereus pasacana): ¿Asociación positiva o dispersión?. Tesis doctoral. Universidad Nacional de Córdoba. Argentina.

de Viana, M.L. 1999. Seed production and seed bank of Trichocereus pasacana (Cactaceae) in northwestern Argentina. Trop. Ecol. 40: 79-84.

Digilio, A.P. \& P. R. Leganame 1982. Los árboles indígenas de la provincia de Tucumán. Opera Lilloana XXXIV. Tucumán. Argentina.

Dimitri, M.J., R.F.J. Leonardis \& J.S. Biolini 1997. El Nuevo libro del árbol. Tercera Edición. Ed. el Ateneo. Buenos Aires. Argentina.

Janzen, D.H. 1969. Seed eaters versus seed size, number, toxicity and dispersal. Evolution 23:1-27.

Janzen, D.H. 1971. Seed predation by animals. Annu. Rev. Ecol. Syst. 2: 465-492.

Janzen, D.H. 1980. Specificity of seed-attacking beetles in a Costa Rican deciduous forest. J. Ecol. 68: 929-952. 
Lee, T. D. \& Bazzaz, FA 1986. Maternal regulation of fecundity: non-random uvule absortion in Cassia fasiculata. Oecologia 68: 459-465.

Mitchell, R. 1977. Bruchid beetles and seed packaging by palo verde. Ecology 58:644-651.

Moles, A.T., D.I. Warton, \& M. Westoby. 2003. Do smallseeded species have higher survival through seed predation than large seeded species? Ecology 84: 3148-3161.

Ortega Baes P., M.L. de Viana \& M. Saravia. 2001. The fate of Prosopis ferox seeds from unremoved pods at Nacional Park Los Cardones. J. Arid Env. 48: 185-190.

Parciak, W. 2002. Seed size, number, and habitat of a fleshy-fruited plant: consequences for seedling establishment. Ecology 83: 794-808.

Schaal, B.A. 1980. Reproductive capacity and seed size in Lupinus texensis. Am. J. Bot. 67: 703-709.
Silvertown J.W. \& J. Lovett Doust 1993. Introduction to Plant population Biology. Blackwell, Londres, Inglaterra.

Stephenson, A.G. 1992. The regulation of maternal investment in plants. In Marshall C. \& J. Grace (Eds.) Fruit and Seed Production. Cambridge, Cambridge, Inglaterra.

Strahler, A.N. 1986. Geografía Física. Omega. Barcelona, España.

Terán, A.L. \& S. Muruaga De L’ Argentier, 1981. Observaciones sobre bruquidos (coleoptera) del noroeste argentino. IV. Estudios morfológicos y biológicos de Amblycerus Hoffmanseggi (Gyll.), Acanthoscelides comptus kingsolver y merobruchus bicoloripes (pic). Acta Zoológica Lilloana XXXVI. Tucumán. Argentina.

Wulff, R.D. 1986. Seed size variation in Desmodium paniculatum. I Factors affecting seed size. J. Ecol. 74: 87-97. 